\title{
Assessment of the Depression, Anxiety, and Stress Scale (DASS-21) in Untreated Obstructive Sleep Apnea (OSA)
}

\author{
University of Western Australia \\ Sergio Starkstein
University of Western Australia
}

Shenooka Nanthakumar and Romola S. Bucks

\author{
Timothy C. Skinner \\ Charles Darwin University
}

David Hillman
Sir Charles Gairdner Hospital, Nedlands,
Western Australia, Australia

Alan James and Michael Hunter

University of Western Australia and Sir Charles Gairdner Hospital, Nedlands, Western Australia, Australia

\begin{abstract}
The assessment of depression in obstructive sleep apnea (OSA) is confounded by symptom overlap. The Depression, Anxiety, and Stress Scale-short form (DASS-21) is a commonly used measure of negative affect, but it not known whether the DASS-21 is suitable for use in an OSA sample. This study compared the fit of Lovibond and Lovibond's (1995) correlated 3-factor structure of the DASS-21 and measurement invariance between a non-OSA and an OSA sample using confirmatory factor analysis. As measurement invariance was not found, to determine the source of non-invariance differential item functioning (DIF) was examined using dMACS. The correlated 3-factor structure (with correlated errors) of the DASS-21 was a better fit in the non-OSA sample. dMACS indicated that there was a degree of DIF for each of the subscales, especially for the Anxiety subscale, in which 2 symptoms (that are also physiological symptoms of OSA) produced lower severity scores in the OSA sample compared with the non-OSA sample. However, the degree of DIF for each of the subscales is not sufficient to cause concern when using the DASS-21; therefore, the total DASS-21 is suitable for use in an OSA sample. Interestingly, the impact of symptom overlap in anxiety symptoms may be reducing anxiety scores because of DIF, which contrasts with the proposed effect of symptom overlap in depression, where it leads to the inflation of depression scores in OSA. This deserves greater consideration in relation to OSA and other clinical disorders or chronic illness conditions with different patterns of overlapping symptoms.
\end{abstract}

\section{Public Significance Statement}

Depression questionnaires assess symptoms that are also found in obstructive sleep apnea (OSA: e.g., fatigue, irritability, sleepiness), which may make questionnaires unsuited to assessing depressive symptoms in individuals with OSA. Evaluation of the Depression, Anxiety, and Stress Scale-short form (DASS-21) reveals that it is a suitable measure to assess depression, anxiety, and stress symptoms in individuals with OSA.

Keywords: depression, obstructive sleep apnea, measurement invariance, negative affect, DASS-21

Shenooka Nanthakumar and Romola S. Bucks, School of Psychology, University of Western Australia; Timothy C. Skinner, School of Psychological and Clinical Sciences, Charles Darwin University; Sergio Starkstein, School of Psychiatry and Clinical Neurosciences, University of Western Australia; David Hillman, West Australian Sleep Disorders Research Institute and Department of Pulmonary Physiology and Sleep Medicine, Sir Charles Gairdner Hospital, Nedlands, Western Australia, Australia; Alan James, School of Medicine and Pharmacology, University of Western Australia and West Australian Sleep Disorders Research Institute, Sir Charles Gairdner Hospital; Michael Hunter, School of Population Health, University of Western Australia and Busselton Population Medical Research Institute, Sir Charles Gairdner Hospital.
The Busselton Healthy Ageing Study was supported by the Office of Science, the Department of Health of the Government of Western Australia, and the Shire of Busselton. The authors thank the Busselton Healthy Ageing Study participants for their contribution to the study. R. S. B., D. H., S. S., A. J., \& M. H. received support from a National Health and Medical Research Council grant (NHMRC 1031575). The contents of the published material are solely the responsibility of the administering institution, a participating institution, or individual authors and do not reflect the views of the NHRMC. S. N. has an Australian Postgraduate Award Scholarship awarded by the University of Western Australia.

Correspondence concerning this article should be addressed to Romola S. Bucks, School of Psychology, University of Western Australia, 35 Stirling Highway, Crawley 6009, Western Australia, Australia. E-mail: romola.bucks@uwa.edu.au 
Obstructive sleep apnea (OSA) is a common and often underdiagnosed condition (Motamedi, McClary, \& Amedee, 2009). It is characterized by recurrent episodes of partial (hypopnea) or a near-complete (apnea) obstruction of the pharyngeal airway during sleep (Spicuzza, Caruso, \& Di Maria, 2015). These obstructive events are associated with drops in oxygen saturation and are usually terminated by arousals, resulting in fragmentation of sleep (Eckert \& Malhotra, 2008; Schroder \& O’Hara, 2005). Epidemiological studies have shown that $13 \%$ of men and $6 \%$ of women have OSA of at least moderate severity (Apnea-Hypopnea Index [AHI] 2:: 15; Peppard et al., 2013). The condition manifests as snoring, nocturnal gasping and choking, excessive daytime sleepiness, reduced quality of life, and fatigue (Gupta, Chandra, Verm, \& Kumar, 2010). OSA also increases the risk of a constellation of health-related issues, including cardiovascular problems, such as hypertension (Marin et al., 2012), stroke (Yaggi et al., 2005), and myocardial infarction (Marin, Carrizo, \& Kogan, 1998), as well as type 2 diabetes (Tasali, Mokhlesi, \& Van Cauter, 2008) and metabolic syndromes (Vgontzas, Bixler, \& Chrousos, 2005).

Previous research (e.g., Harris, Glozier, Ratnavadivel, \& Grunstein, 2009; Schroder \& O’Hara, 2005; Nanthakumar, Bucks, \& Skinner, 2016) has highlighted the association between OSA and depression. Cardinal symptoms of depression include low mood, anhedonia, hypersomnia/insomnia, fatigue/loss of energy, feelings of worthlessness/guilt, concentration difficulty/indecisiveness, psychomotor agitation/retardation, suicidal ideation, appetite changes, and weight gain/loss (Diagnostic and Statistical Manual of Mental Disorders [Fifth edition, DSM-5]; American Psychiatric Association, 2013). In addition, depression frequently presents with symptoms of anxiety and stress, such as excessive worry, phobia, and irritability (DSM-5; American Psychiatric Association, 2013). Depression questionnaires are commonly used to assess these different features of depression in clinical and research settings (Coyne, Thompson, Klinkman, \& Nease Jr., 2002). The prevalence rates of current depression in OSA when using depression questionnaires (using standardized cutoffs) in clinicalbased and population-based studies have ranged from 8\% to 68\% (Nanthakumar et al., 2016). The assessment of depression in OSA is confounded by the overlap in symptoms between the two conditions, such as insomnia, fatigue, decreased libido, irritability, weight changes, and cognitive difficulties (Harris et al., 2009; Nanthakumar et al., 2016). Different depression questionnaires have varying proportions of shared symptoms, and increasing symptom overlap is associated with increasing prevalence of depression in OSA (Nanthakumar et al., 2016). This overlap in symptomatology makes it difficult to determine which symptoms reliably identify depression in the presence of OSA.

The Depression, Anxiety, and Stress Scale (DASS; Lovibond \& Lovibond, 1995) is a frequently used measure of overall negative affect in clinical and non-clinical populations. The DASS contains three subscales that assess depression, anxiety, and stress symptoms, and it is available in the original 42-item (14 items per subscale) and a shortened 21-item form (DASS-21; 7 items per subscale). As previously noted, anxiety and stress symptoms are associated features of depression. Previous studies have shown that the scale has sound psychometric properties of a correlated, threefactor structure (depression, anxiety, and stress) across clinical and non-clinical samples (Antony, Bieling, Cox, Enns, \& Swinson, 1998; Henry \& Crawford, 2005), including other chronic disease samples (e.g., persistent pain; Wood, Nicholas, Blythe, Asghari, \& Gibson, 2010).

Measurement invariance assesses the equivalence of the factor structure parameters (i.e., factor structure, factor loadings, factor means, item loadings) across different samples (Byrne \& Watkins, 2003; Nye, Roberts, Saucier, \& Zhou, 2008; Steenkamp \& Baumgartner, 1998). In the absence of measurement invariance, comparisons on a measure are problematic and ambiguous. That is, one cannot determine if an observed difference between samples represents a true difference in the underlying construct or systematic biases in the way people from different samples perceive and respond to items (Byrne \& Watkins, 2003; Cheung \& Rensvold, 2002; Horn \& McArdle, 1992). When examining the symptoms assessed in the DASS-21 in an OSA sample, there are two underlying factors predicting a person's score: (a) the degree to which there are true differences in the way that the person is genuinely depressed/anxious/stressed and (b) the degree to which they are also endorsing items as a function of their OSA. Therefore, questionnaires that consist of a high proportion of overlapping symptoms with OSA may not be assessing genuine depression/anxiety/ stress in OSA.

In a recent meta-analysis by Nanthakumar et al. (2016), symptoms of depression in commonly used depression scales were categorized into overlapping and non-overlapping symptoms. These categories can also be used to divide the DASS-21 items. Non-overlapping symptoms of depression reflect anhedonia (e.g., "I felt that I had nothing to look forward to") or depressive cognitions (e.g., "I felt I was not worth much as a person"). Non-overlapping symptoms of anxiety assess anxious cognitions (e.g., "I felt scared without any good reason") and non-overlapping stress symptoms assess stress cognitions (e.g., "I felt that I was using a lot of nervous energy") and agitation/restlessness symptoms (e.g., "I found myself getting agitated"). By contrast, the Anxiety subscale assesses overlapping somatic symptoms (e.g., "I was aware of dryness of my mouth" and "I experienced breathing difficulty” [e.g., excessively rapid breathing, breathlessness in the absence of physical exertion]), and the Stress subscale assesses overlapping irritability symptoms (e.g., "I felt that I was rather touchy" and "I was intolerant of anything that kept me from getting on with what I was doing”), but the Depression subscale contains no overlapping symptoms. No study to date has examined the impact of overlapping symptoms on the suitability of the total DASS-21 in an OSA sample compared with a non-OSA sample.

The present study examined (a) if Lovibond and Lovibond's (1995) correlated three-factor structure holds in an OSA sample compared with a non-OSA sample, and (b) measurement invariance across these samples. We hypothesized that there may be a lack of measurement invariance across samples, primarily due to differential fit in the Anxiety and/or Stress subscales, because of symptom overlap.

\section{Method}

\section{Participants and Protocol}

Participants were selected from the Busselton Healthy Ageing Study. Busselton is a coastal community in the southwest of Western Australia with a relatively stable population of predominantly European descent (James et al., 2013). All noninstitution- 
alized adults born from 1946 to 1964, aged 45-66 years at the time of the baseline survey, who currently live in the Shire and are listed on the electoral roll, are eligible to participate. Data were obtained from Phase 1 of this longitudinal study. Informed consent was obtained from each participant, who completed a comprehensive medical and lifestyle questionnaire (e.g., demographics and questions about mood, sleep history, smoking history, alcohol consumption, and presence of chronic diseases) and physical health assessments. Sleep apnea screening devices were offered to participants to use over one night at home. Participants who received current continuous positive airway pressure (CPAP) or mandibular advancement splint therapy for sleep apnea were excluded from the sleep apnea screening study. Sleep studies were conducted using dual-channel (oximetry and nasal pressure) ApneaLink devices (Resmed, Sydney, Australia). Participants were instructed on how to fit and operate the units, and the monitors were returned to the study center the following day. Data were downloaded and automatically analyzed using ApneaLink software version 8.00. Scores of respiratory disturbances including AHI, number of snoring events, and oxygen desaturation were recorded. The ApneaLink has been found to be a good screening tool for OSA (Crowley et al., 2013; Erman, Stewart, Einhorn, Gordon, \& Casal, 2007; Gantner et al., 2010; Ng et al., 2009). An AHI of 2::15 on an ApneaLink has adequate sensitivity (66.7-100.0\%) and specificity (88.5-100.0\%) in indicating the presence of OSA compared with full polysomnography (PSG; see Crowley et al., 2013; Erman et al., 2007; Ng et al., 2009; Ragette, Wang, Weinreich, \& Teschler., 2010). The study received approval from the University of Western Australia Human Research Ethics Committee.

\section{OSA Sample}

There were 2,129 individuals recruited at baseline who completed an overnight sleep study, of whom 285 (13.39\%) had an AHI of 2::15 using the ApneaLink, and reported no other sleep disorder (based on self-reported medical history). The mean age was 58 years $(S D=5.26$; range $=45-68$ years $)$, mean body mass index $(\mathrm{BMI})$ was $31.03(S D=6.12$; range $=17.70-65.70)$, and mean AHI was 24.68 ( $S D=11.75$; range 15-89). One hundred and nine (38.25\%) individuals were female.

\section{Age- and Gender-Matched Controls (Non-OSA Sample)}

From the remaining individuals whose AHI was $<15$ and who reported nosleep disorder, 285(15.46\%) age-and gender-matched individuals were selected. These individuals were selected by pairing an OSA participant to a non-OSA participant who was of the same gender and age \pm 5 years of the age of the OSA participant. The mean age was 58 years $(S D=5.24$; range $=45-68$ years), mean BMI was $30.64(S D=4.20$; range $=21.87-44.42)$, and mean AHI was 5.16 ( $S D=3.99$; range: $0-14$ ). The AHI was $<5$ in $59.30 \%$ and $<10$ in $88.10 \%$ of individuals, and 109 (38.25\%) were female. There was no significant difference in BMI or age between the two samples $(p>.05)$. As expected, AHI was significantly lower in the age- and gender-matched controls $(p<.01)$.

\section{Materials}

The DASS-21 (Lovibond \& Lovibond, 1995) is a 21-item selfreport questionnaire designed to measure depression, anxiety, and stress symptoms. It is the short form of Lovibond and Lovibond's (1995) 42-item version, from which the 21 highest loading items were selected ( 7 from each of the 3 subscales). Participants indicate the degree to which they felt negative affect symptoms over the past week, from 0 (did not apply to me at all) to 3 (applied to me very much, or most of the time). Scale scores range from 0 to 21 , but are doubled so as to be comparable to the 42-item scale, with higher scores indicating greater depression, anxiety, and stress, respectively. The subscales have good internal reliability (Depression subscale: Cronbach's $\mathrm{a}=.82$ to .84; Anxiety subscale: Cronbach's $\mathrm{a}=.74$ to .81 ; Stress subscale: Cronbach's $\mathrm{a}=$ .74 to .88; Norton, 2007) as well as good convergent and discriminant validity (Henry \& Crawford, 2005), and the full scale has a reliable correlated three-factor structure in clinical and nonclinical samples (Antony et al., 1998; Henry \& Crawford, 2005).

\section{Data Analysis Plan}

Data screening and descriptive analyses were performed using SPSS 19.0 for Windows, IBM (2015B). Confirmatory factor analysis (CFA) and measurement invariance analyses were performed using MPlus, version 3.0 (Muthén \& Muthén, 1998-2011) and dMACS (Nye \& Drasgow, 2011).

CFA. CFA was used to examine Lovibond and Lovibond's (1995) correlated three-factor structure of the DASS-21 in the OSA and non-OSA samples. As recommended by Muthén and Muthén (1998-2011), we used maximum likelihood estimation with robust standard errors because this is robust to non-normality. $x$ was used to evaluate overall model fit, along with the comparative fit index (CFI; Bentler, 1990), Tucker-Lewis Index (TLI; Tucker \& Lewis, 1973), root-mean-square error of approximation (RMSEA; Steiger \& Lind, 1980), and standardized root mean squared residual (SRMR; Jöreskog \& Sörbom, 1998). Recommended cutoffs for the fit indexes were CFI: 2::.95, TLI: 2::.95, RMSEA: <.050, and SRMR: <.060 (Hu \& Bentler, 1999).

Measurement invariance. Provided that the correlated threefactor structure of the DASS-21 was an acceptable fit in the nonOSA sample, the multigroup confirmatory factor analysis (MGCFA) approach for testing measurement invariance was conducted by performing configural, metric, and then scalar invariance testing (Cheung \& Rensvold, 2002; Vandenberg \& Lance, 2000). A $x^{2}$ difference test using scaling correction factors for MLR across unconstrained and a series of constrained measurement models was performed to examine the different levels of measurement invariance (Muthén \& Muthén, 1998-2011). The unconstrained model is estimated without any conditions, whereas the constrained models are estimated with the condition that one or more specified factor parameters would have the same value for both samples (Muthén \& Muthén, 1998-2011). A lack of measurement invariance is indicated by a statistically significant $x^{2}$ difference between the respective pair of models (Muthén \& Muthén, 1998-2011) and a LICFI of >.002 (Meade, Johnson, \& Braddy, 2008).

Configural invariance was tested first, which evaluates whether the same overall factor structure holds in the two samples (Cheung \& Rensvold, 2002; Milfont \& Fischer, 2010; 
Vandenberg \& Lance, 2000). Configural invariance is a prerequisite for higher levels of equivalence testing (i.e., metric and scalar invariance). If configural invariance was met, then metric invariance and scalar invariance were tested. Metric invariance evaluates the degree to which the factor loadings are equal across samples, and scalar invariance assesses the equality of intercept terms (i.e., means/thresholds) between the samples (Cheung \& Rensvold, 2002; Milfont \& Fischer, 2010; Vandenberg \& Lance, 2000).

If measurement non-invariance was found, then CFA mean and covariance structure (MACS) analysis to examine the source of noninvariance by assessing DIF was conducted using dMACS (see Nye \& Drasgow, 2011), evaluating (a) which items are being responded to in a different way between the samples and (b) the magnitude of these differences between the samples (i.e., DIF). Effect sizes were compared to Cohen's (1988) guidelines $(0.20=$ small, $0.50=$ medium, $0.80=$ large $)$.

\section{Results}

\section{Descriptive Statistics}

Means, SDs, ranges, a values, and difference in means for the three subscale scores for each sample are shown in Table 1.

\section{CFA}

The correlated three-factor structure (Model 1a) had fairly poor fit (see Table 2). In theory, the requirement for any item to load exclusively on a single latent construct has been problematic in CFA because items within a measure can sometimes be multidimensional (see Morin, Arens, \& Marsh, 2016), which may account for inadequate fit of the correlated three-factor model. Given that work from Nanthakumar et al. (2016) and Fried (2015) suggests that depression, anxiety, and stress symptoms are multidimensional and may cluster into subfacets (e.g., anhedonia, cognition, cognitive symptoms), we allowed error terms within subscales to correlate. As in Szabó (2010), correlations were based on modification indices (MIs) supported by a conceptual rationale, restricting correlated errors to item pairs within a subscale. Correlating error terms to improve model fit was recommended by Henry and Crawford (2005) and has been applied in other studies (e.g., Asghari, Saed, \& Dibajnia, 2008; Szabó, 2010; Wood et al., 2010) that have examined the factor structure of the DASS-21. Correlated pairs included four Depression item pairs reflecting anhedonia or cognition symptoms (range of MIs = 4.03-47.20), six item pairs from the Anxiety subscale reflecting consequences of panic/anxiety (range of MIs = 6.12-13.54), and one item pair from the Stress subscale reflecting restlessness (range of $\mathrm{MI}=9.32$ ). Permitting correlated errors (see Table 3) produced a well-fitting model (Model 1b, Table 2). When this model was then tested with the OSA sample (Model 2, Table 2), fit statistics indicated less than ideal fit according to the significant $x^{2}$, TLI, and CFI; therefore, we proceeded to invariance testing.

\section{Measurement Invariance}

Model 1b served as the baseline model in evaluating measurement invariance across samples. Results from configural invariance analysis indicated that the LIX $x^{2}$ was significant $(p<.001)$ and LICFI $=.003$. Therefore, further invariance analyses were not conducted.

Given the lack of configural invariance, dMACS was used to investigate the source of measurement non-invariance by assessing DIF. MPlus estimates of the item parameters that were used to calculate the effect sizes for the Depression, Anxiety, and Stress subscales are provided in Table 3. The first column shows the effect sizes of non-equivalence for each of the items (dMACS), and the last four columns show the factor loadings and the intercepts for the OSA and non-OSA samples.

A range of non-equivalence was found in the subscales. The broadest range of effect sizes was observed for the Anxiety subscale, in which effects ranged from very small (e.g., 0.12 for the item Scared without reason) to medium (e.g., 0.57 for the item Action of my heart in the absence of physical exertion). Inspection of the loadings and intercepts indicated that the items Dryness in mouth and Action of my heart in the absence of physical exertion produced the largest differences between the two samples, particularly at the upper end of the latent trait continuum (see Nye \& Drasgow, 2011). Figure 1 illustrates that the impact of DIF on the scores for these two items (1a. Dryness of mouth; 1b. Action of my heart) contrasted with a third item (1c. Worry about panic and making a fool of self), which had an effect size of 0.31 . For the first two items, at higher levels of anxiety (the latent trait), individuals with OSA endorsed a lower severity score than those without OSA. That is, DIF produced lower responses on the subscale item for similar levels of the latent trait of anxiety for the OSA sample than the non-OSA sample. For the third item illustrated, the effect was the opposite: OSA individuals with higher levels of anxiety scored higher on that item than those without OSA. Inspection of the pattern of loadings across the anxiety items revealed that four had higher loadings for the non-OSA sample and two for the OSA sample. A small range of non-equivalence was identified in the

Table 1

Characteristics of the DASS-21 Subscales for the Non-OSA and OSA Samples

\begin{tabular}{|c|c|c|c|c|c|c|c|c|c|}
\hline \multirow[b]{2}{*}{ Subscale } & \multicolumn{4}{|c|}{ Non-OSA sample $(N=285)$} & \multicolumn{4}{|c|}{ OSA sample $(N=285)$} & \multirow{2}{*}{$\begin{array}{l}\text { Difference in } \\
\text { means between } \\
\text { samples }\end{array}$} \\
\hline & $M$ & $S D$ & Range & $\mathrm{a}$ & $M$ & $S D$ & Range & $\mathrm{a}$ & \\
\hline Depression & 3.36 & 4.59 & $0-24$ & .83 & 4.17 & 5.91 & $0-34$ & .87 & $p>.05$ \\
\hline Anxiety & 2.25 & 3.44 & $0-26$ & .68 & 2.84 & 3.94 & $0-18$ & .66 & $p>.05$ \\
\hline Stress & 5.75 & 6.16 & $0-34$ & .86 & 6.74 & 7.00 & $0-34$ & .88 & $p>.05$ \\
\hline
\end{tabular}

Note. $\quad$ DASS-21 = Depression, Anxiety, and Stress Scale; OSA = Obstructive Sleep Apnea. Scores on the DASS-21 are multiplied by 2 to calculate the final subscale scores, producing a maximum of 42 points. 
Table 2

Lovibond and Lovibond's (1995) Correlated Three-Factor Structure (With Correlated Errors) of the DASS-21 CFA in the Non-OSA and OSA Samples

\begin{tabular}{|c|c|c|c|c|c|c|c|c|c|}
\hline Model & $d f$ & $x^{2}$ & $p$ & SRMR & CFI & TLI & RMSEA & LO 90 & HI 90 \\
\hline 1a. Non-OSA sample: Correlated three-factor structure & 186 & 314.31 & $<.001$ & .060 & .90 & .88 & .049 & .040 & .058 \\
\hline $\begin{array}{l}\text { 1b. Non-OSA sample: Correlated three-factor structure } \\
\text { (with correlated errors) }\end{array}$ & 175 & 197.55 & .117 & .049 & .98 & .98 & .021 & 0 & .035 \\
\hline $\begin{array}{l}\text { 2. OSA sample: Correlated three-factor structure (with } \\
\text { correlated errors) }\end{array}$ & 175 & 271.22 & $<.001$ & .049 & .94 & .93 & .044 & .033 & .054 \\
\hline
\end{tabular}

Note. DASS-21 = Depression, Anxiety, and Stress Scale; OSA = Obstructive Sleep Apnea; LO $90=$ Lower boundary of $90 \%$ confidence interval; HI $90=$ Upper boundary of $90 \%$ confidence interval. Recommended cutoffs for the fit indexes: SRMR <.060, CFI 2::.95, TLI 2::.95, and RMSEA <.050(Hu \& Bentler, 1999).

Depression and Stress subscales, but there were no meaningful differences in the way DIF affected the way these items were being responded to between the samples.

Collapsing across all of the items within each subscale revealed only small effects of DIF on the scales' properties (see Table 4). The largest difference in mean was -0.49 points for the Stress subscale and the smallest, despite the effects previously described, was -0.29 points for the Anxiety subscale. The negative values in this column suggest that DIF results in higher means for the OSA sample. However, these differences were in raw score points; therefore, compared with the non-OSA sample, the OSA sample had means that were 0.39 points higher for the Depression subscale, 0.29 points higher for the Anxiety subscale, and 0.49 points higher for the Stress subscale because of DIF. Because these differences were at the scale level, they must be interpreted relative to a 42-point maximum score (i.e., seven items with four response options coded 0 to 3, doubled to 42), which indicates that they are small. DIF accounted for $97.5 \%, 100 \%$, and $98 \%$ of the observed

Table 3

Item Parameters for the Depression, Anxiety, and Stress Subscales

\begin{tabular}{|c|c|c|c|c|c|}
\hline \multirow[b]{2}{*}{ Subscale, (item number), and item name } & \multirow[b]{2}{*}{ dMACS } & \multicolumn{2}{|c|}{ Factor loading } & \multicolumn{2}{|c|}{ Intercept } \\
\hline & & Non-OSA sample & $\overline{\text { OSA sample }}$ & Non-OSA sample & OSA sample \\
\hline \multicolumn{6}{|l|}{ Depression subscale } \\
\hline (3) Positive feelings & .30 & 0.76 & 0.91 & .22 & .31 \\
\hline (5) Initiative 1,2 & .09 & 0.88 & 0.88 & .51 & .56 \\
\hline (10) Look forward to ${ }^{1}$ & .25 & 0.89 & 0.73 & .16 & .18 \\
\hline (13) Down-hearted and blue ${ }^{a}$ & .20 & 1.00 & 1.00 & .29 & .36 \\
\hline (16) Enthusiastic 2,3 & .22 & 0.98 & 1.01 & .31 & .39 \\
\hline (17) Self-worth ${ }^{4}$ & .21 & 0.66 & 0.70 & .13 & .19 \\
\hline (21) Life was meaningless 3,4 & .11 & 0.45 & 0.42 & .07 & .09 \\
\hline Latent mean* & & 0 & 0 & & \\
\hline Latent variance & & 0.14 & 0.23 & & \\
\hline \multicolumn{6}{|l|}{ Anxiety subscale } \\
\hline (2) Dryness of mouth & .47 & 1.41 & 0.45 & .37 & .49 \\
\hline (4) Breathing difficulty ${ }^{5,6,7,8,9}$ & .18 & 1.12 & 0.92 & .13 & .12 \\
\hline (7) Trembling ${ }^{5}$ & .18 & 0.47 & 0.66 & .09 & .12 \\
\hline (9) Worry about panic and making a fool of self ${ }^{6,10}$ & .31 & 1.10 & 1.45 & .19 & .24 \\
\hline (15) Close to panic ${ }^{7}$ & .23 & 0.94 & 0.91 & .07 & .14 \\
\hline (19) Action of my heart ${ }^{8}$ & .57 & 1.58 & 0.71 & .20 & .21 \\
\hline (20) Scared $\mathrm{d}^{\mathrm{a}, 9,10}$ & .12 & 1.00 & 1.00 & .08 & .11 \\
\hline Latent mean* & & 0 & 0 & & \\
\hline Latent variance & & 0.03 & 0.08 & & \\
\hline \multicolumn{6}{|l|}{ Stress subscale } \\
\hline (1) Wind down ${ }^{11}$ & .16 & 0.79 & 0.92 & .53 & .60 \\
\hline (6) Overreact & .17 & 1.06 & 0.95 & .52 & .59 \\
\hline (8) Nervous energy & .20 & 0.62 & 0.70 & .22 & .29 \\
\hline (11) Agitated & .22 & 1.04 & 0.92 & .42 & .48 \\
\hline (12) Difficult to relax ${ }^{a, 11}$ & .21 & 1.00 & 1.00 & .40 & .50 \\
\hline (14) Intolerant & .20 & 0.83 & 0.93 & .40 & .48 \\
\hline (18) Touchy & .15 & 0.95 & 0.93 & .38 & .44 \\
\hline Latent mean ${ }^{*}$ & & 0 & 0 & & \\
\hline Latent variance & & 0.20 & 0.26 & & \\
\hline
\end{tabular}

Note. OSA = Obstructive Sleep Apnea; MACS $=$ mean and covariance structure. dMACS values are effect sizes: $0.20=$ small, $0.50=$ medium, $0.80=$ large.

${ }^{a}$ Indicates referent item. ${ }^{*}$ Latent means were set to 0 on dMACS (see Nye \& Drasgow, 2011). Factor loadings are unstandardized. Subscript numbers that are the same within each subscale indicate that the error term for these item pairs were permitted to correlate. 

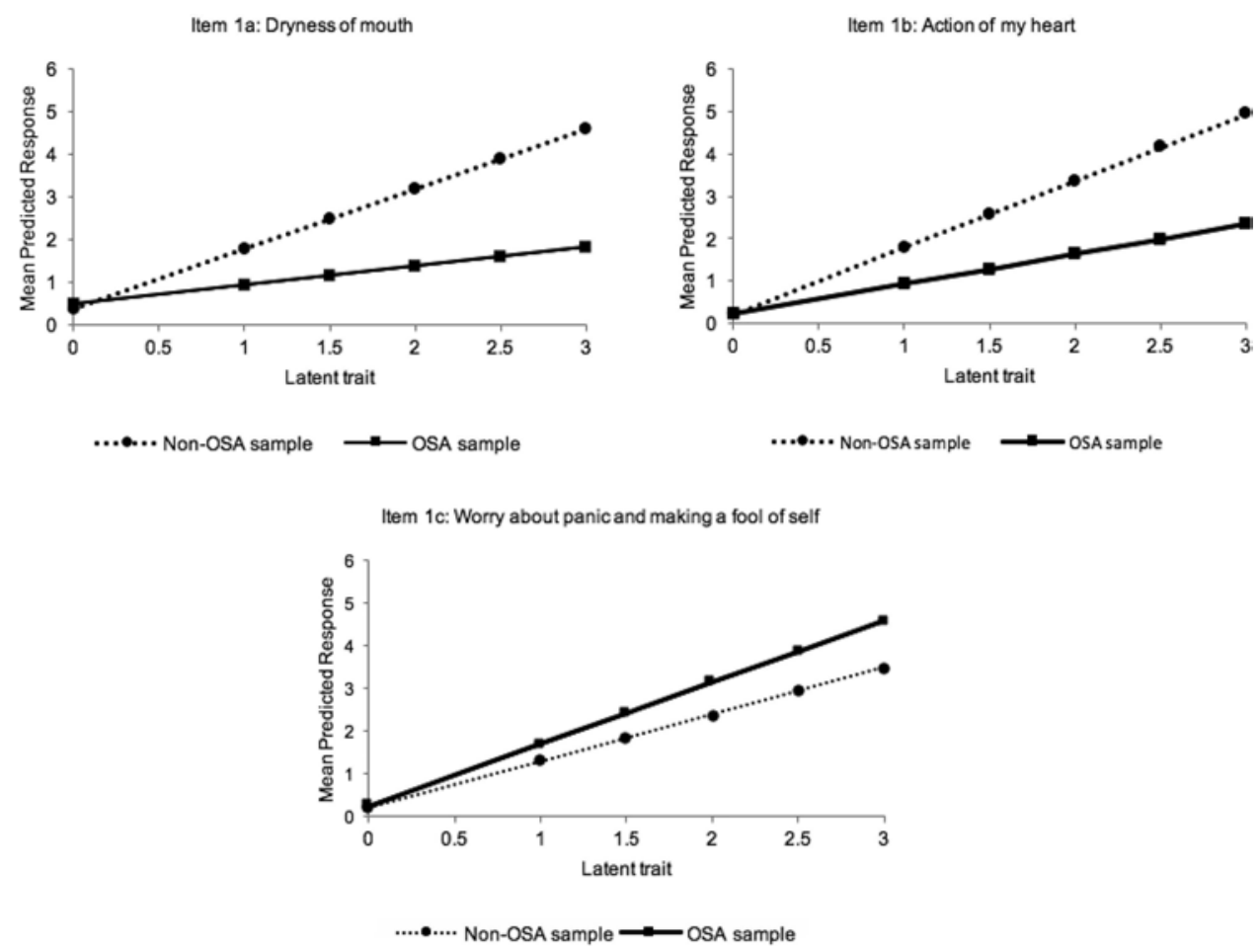

Figure 1. Comparing the items (1a) Dryness of mouth, (1b) Action of my heart, and (1c) Worry about panic and making a fool of self, across the OSA and non-OSA samples.

differences in means between the samples on the Depression, Anxiety, and Stress subscales, respectively. Therefore, the majority of the observed differences between the means of the two samples were due to DIF, rather than true mean differences, but the overall effect on scale mean scores was negligible.

In regards to the variance of the subscales, in the most extreme case, nonequivalence accounted for variance 1.67 points higher on the Anxiety subscale in the OSA sample than the non-OSA sample. In addition, DIF accounted for $0 \%$, $135.77 \%$, and $9.34 \%$ of the observed differences in variance among the samples on the Depression, Anxiety, and Stress subscales, respectively. Therefore, the observed differences between the variances of the two samples on the Anxiety subscale were likely due to DIF, albeit the variances were not large to begin with.

\section{Discussion}

The present study explored Lovibond and Lovibond's (1995) correlated three-factor structure of the DASS-21 in OSA and non-OSA samples by means of CFA and examined measurement invariance. The results showed that Lovibond and Lovibond's (1995) correlated three-factor structure did not fit the OSA sample as well as the non-OSA sample. In addition, configural invariance was not found, suggesting that both samples may be differently interpreting the constructs of the DASS-21 (Cheung \& Rensvold, 2002; Milfont \& Fischer, 2010). Inspection of the individual items within the subscales to determine the source of this difference indicated that overall, DIF led to slightly higher factor means and variances for each of the subscales in the OSA sample compared with the non-OSA sample. However, the actual difference that this

Table 4

Measurement Equivalence of the Depression, Anxiety, and Stress Subscales Between the Non-OSA Sample (Referent Group) and OSA Sample (Focal Group)

\begin{tabular}{|c|c|c|c|c|c|c|c|}
\hline Subscale & LIMean & $\begin{array}{l}\text { Observed mean } \\
\text { difference }\end{array}$ & $\begin{array}{l}\% \text { of observed mean } \\
\text { difference }\end{array}$ & LIVar & $\begin{array}{l}\text { Differences in } \\
\text { observed variance }\end{array}$ & $\begin{array}{l}\text { \% of observed } \\
\text { differences }\end{array}$ & $\begin{array}{l}\text { dMACS min } \\
\text { to max }\end{array}$ \\
\hline Depression & -0.39 & -0.40 & 97.5 & 0.03 & -3.37 & 0 & .09 to .30 \\
\hline Anxiety & -0.29 & -0.29 & 100 & -1.67 & -1.23 & 135.77 & .11 to .57 \\
\hline Stress & -0.49 & -0.50 & 98 & 0.24 & -2.57 & 9.34 & .15 to .22 \\
\hline
\end{tabular}

Note. OSA = Obstructive Sleep Apnea; DIF = differential item functioning; LIM = amount of observed mean differences that can be attributed to DIF (referent group - focal group). Observed mean difference $=$ DIF + true mean differences. LIVar $=$ difference in the variances of the subscale due to DIF. Differences in observed variance = DIF + true variances. For all difference metrics, negative values indicate larger values in the OSA sample than in the non-OSA sample. 
produced in mean subscale scores was small (between just 0.29 and 0.49 of a point; i.e., less than half of a point across any subscale overall). Therefore, on balance, we take the view that the degree of DIF for each of the Depression, Anxiety, and Stress subscales of the DASS-21 is not sufficient to cause concern when using the full-scale DASS-21 to assess negative affect in an OSA sample.

It is interesting to note that there was no impact of the symptom overlap within the Stress subscale. However, inspection of the problematic items within the Anxiety subscale does challenge our understanding of the impact of symptom overlap on the measurement of negative affect in clinical disorders or chronic illness. Although the mean of the Anxiety subscale differed only by 0.29 of a point, there were modest problems with two symptoms within this subscale. For two physiological symptoms of anxiety (items: Dryness in mouth, and Action of my heart in the absence of physical exertion), small to medium-sized differences in the way these items were endorsed were evident, particularly at the upper end of the latent trait continuum. That is, highly anxious respondents in the OSA and non-OSA samples responded differently to these items. Although dryness of the mouth was identified as an overlapping symptom with OSA, heart action was not categorized as an overlapping symptom in Nanthakumar et al. (2016). However, on reflection, OSA is associated with increased risk of atrial fibrillation (see Gami et al., 2007), which may be experienced as heartbeat changes in the absence of exertion. Strikingly, the effect of symptom overlap for these items produced lower severity scores in the OSA sample, despite higher mean subscale scores. This contrasts markedly with the evidence that overlapping symptoms of depression and OSA lead to inflation of depression scores, thus producing higher prevalence estimates of depression in OSA (Nanthakumar et al., 2016). Therefore, the impact of symptom overlap on depressive symptomology may have separate, and opposing effects, depending on the nature of the symptom and the clinical disorder or chronic illness. Critically, however, from the point of view of each DASS-21 subscale, these opposing effects appear to cancel each other out, making the DASS-21 suitable for use in OSA in its entirety.

The present study is the first to examine whether the fullscale of the DASS-21 is suitable for use in OSA and examine the impact of symptom overlap. Although individuals were recruited from an epidemiological sample, there was a limited age range because the sample consisted of baby boomers who were on the electoral roll. It is possible that there may be differential effects in measurement invariance in younger or older age groups (Gabbay \& Lavie, 2012). Furthermore, although an ApneaLink has been found to have adequate sensitivity and specificity as a screening tool for diagnosing OSA, overnight PSG, which is the gold standard for diagnosing OSA, was not available (Mansfield, Antic, \& McEvoy, 2013; Ragette et al., 2010). In addition, because individuals in the non-OSA sample were matched to the OSA sample on age, gender, and as close as possible to BMI, we had insufficient participants to select non-OSA controls with an AHI of $<10$ or even $<5$. That said, $88 \%$ of the non-OSA sample had an AHI of $<10$ and $59 \%$ had an AHI of $<5$. Future studies may wish to recruit a wide age range, diagnose OSA with PSG, and use a lower AHI cutoff (e.g., AHI <5) to select individuals in the non-OSA sample to further examine any difference between OSA and non-OSA samples and to examine measurement invariance.

Although this study suggests that the full-scale DASS-21 is suitable for use in OSA, this is clearly an area that warrants greater consideration in relation to OSA; with respect to other negative affect measures that may suffer greater DIF; and in other clinical disorders or chronic illness conditions such as insomnia (Carney, Ulmer, Edinger, Krystal, \& Knauss, 2009), Parkinson's disease (Schrag et al., 2007), and diabetes (Anderson, Freedland, Clouse, \& Lustman, 2001) because DIF may impact the suitability of other questionnaires used to assess negative affect in these conditions by inflating or reducing the degree to which symptoms are endorsed in unexpected or confounding ways.

\section{References}

American Psychiatric Association. (2013). Diagnostic and statistical manual of mental disorders (5th ed.). Arlington, VA: Author.

Anderson, R. J., Freedland, K. E., Clouse, R. E., \& Lustman, P. J. (2001). The prevalence of comorbid depression in adults with diabetes: A meta-analysis. Diabetes Care, 24, 1069-1078. http://dx.doi.org/10 .2337/diacare.24.6.1069

Antony, M. M., Bieling, P. J., Cox, B. J., Enns, M. W., \& Swinson, R. P. (1998). Psychometric properties of the 42-item and 21-item versions of the Depression Anxiety Stress Scales in clinical groups and a community sample. Psychological Assessment, 10, 176-181. http://dx.doi.org/10 $.1037 / 1040-3590.10 .2 .176$

Asghari, A., Saed, F., \& Dibajnia, P. (2008). Psychometric properties of the Depression Anxiety Stress Scales-21 (DASS-21) in a non-clinical Iranian sample. International Journal of Psychology, 2, 82-102.

Bentler, P. M. (1990). Comparative fit indexes in structural models. Psychological Bulletin, 107, 238-246. http://dx.doi.org/10.1037/0033-2909 107.2.238

Byrne, B. M., \& Watkins, D. (2003). The issue of measurement invariance revisited. Journal of Cross-Cultural Psychology, 34, 155-175. http://dx doi.org/10.1177/0022022102250225

Carney, C. E., Ulmer, C., Edinger, J. D., Krystal, A. D., \& Knauss, F. (2009). Assessing depression symptoms in those with insomnia: An examination of the Beck Depression Inventory 2nd ed. (BDI-II). Journal of Psychiatric Research, 43, 576-582.

Cheung, G. W., \& Rensvold, R. B. (2002). Evaluating goodness-of-fit indexes for testing measurement invariance. Structural Equation Modeling, 9, 233-255. http://dx.doi.org/10.1207/S15328007SEM0902_5

Cohen, J. (1988). Statistical power analysis for the behavioral sciences (2nd ed.). Hillsdale, NJ: Erlbaum.

Coyne, J. C., Thompson, R., Klinkman, M. S., \& Nease, D. E., Jr. (2002). Emotional disorders in primary care. Journal of Consulting and Clinical Psychology, 70, 798-809. http://dx.doi.org/10.1037/0022-006X.70.3 .798

Crowley, K. E., Rajaratnam, S. M., Shea, S. A., Epstein, L. J., Czeisler, C. A., \& Lockley, S. W., \& the Harvard Work Hours, Health and Safety Group. (2013). Evaluation of a single-channel nasal pressure device to assess obstructive sleep apnea risk in laboratory and home environments. Journal of Clinical Sleep Medicine, 9, 109-116.

Eckert, D. J., \& Malhotra, A. (2008). Pathophysiology of adult obstructive sleep apnea. Proceedings of the American Thoracic Society, 5, 144-153. http://dx.doi.org/10.1513/pats.200707-114MG

Erman, M. K., Stewart, D., Einhorn, D., Gordon, N., \& Casal, E. (2007). Validation of the ApneaLink for the screening of sleep apnea: A novel and simple single-channel recording device. Journal of Clinical Sleep Medicine, 3, 387-392. 
Fried, E. I. (2015). Problematic assumptions have slowed down depression research: Why symptoms, not syndromes are the way forward. Frontiers in Psychology, 6, 309. http://dx.doi.org/10.3389/fpsyg.2015.00309

Gabbay, I. E., \& Lavie, P. (2012). Age- and gender-related characteristics of obstructive sleep apnea. Sleep and Breathing, 16, 453-460. http://dx .doi.org/10.1007/s11325-011-0523-z

Gami, A. S., Hodge, D. O., Herges, R. M., Olson, E. J., Nykodym, J., Kara, T., \& Somers, V. K. (2007). Obstructive sleep apnea, obesity, and the risk of incident atrial fibrillation. Journal of the American College of Cardiology, 49, 565-571. http://dx.doi.org/10.1016/j.jacc.2006.08.060

Gantner, D., Ge, J. Y., Li, L. H., Antic, N., Windler, S., Wong, K., . . . McEvoy, D. (2010). Diagnostic accuracy of a questionnaire and simple home monitoring device in detecting obstructive sleep apnoea in a Chinese population at high cardiovascular risk. Respirology, 15, 952960. http://dx.doi.org/10.1111/j.1440-1843.2010.01797.x

Gupta, R. K., Chandra, A., Verm, A. K., \& Kumar, S. (2010). Obstructive sleep apnoea: A clinical review. The Journal of the Association of Physicians of India, 58, 438-441.

Harris, M., Glozier, N., Ratnavadivel, R., \& Grunstein, R. R. (2009). Obstructive sleep apnea and depression. Sleep Medicine Reviews, 13, 437-444. http://dx.doi.org/10.1016/j.smrv.2009.04.001

Henry, J. D., \& Crawford, J. R. (2005). The short-form version of the Depression Anxiety Stress Scales (DASS-21): Construct validity and normative data in a large non-clinical sample. British Journal of Clinical Psychology, 44, 227-239. http://dx.doi.org/10.1348/014466505X29657

Horn, J. L., \& McArdle, J. J. (1992). A practical and theoretical guide to measurement invariance in aging research. Experimental Aging Research, 18, 117-144. http://dx.doi.org/10.1080/03610739208253916

Hu, L. T., \& Bentler, P. M. (1999). Cutoff criteria for fit indexes in covariance structure analysis: Conventional criteria versus new alternatives. Structural Equation Modeling: A Multidisciplinary Journal, 6, $1-55$.

James, A., Hunter, M., Straker, L., Beilby, J., Bucks, R., Davis, T., . . . the Busselton Healthy Ageing Study Investigator Group. (2013). Rationale, design and methods for a community-based study of clustering and cumulative effects of chronic disease processes and their effects on ageing: The Busselton healthy ageing study. BMC Public Health, 13, 936. http://dx.doi.org/10.1186/1471-2458-13-936

Jöreskog, K. G., \& Sörbom, D. (1998). LISREL 8: Structural equation modeling with the SIMPLIS command language. Hillsdale, NJ: Lawrence Erlbaum.

Lovibond, S. H., \& Lovibond, P. F. (1995). Manual for the depression anxiety stress scales. Sydney, New South Wales, Australia: Psychology Foundation.

Mansfield, D. R., Antic, N. A., \& McEvoy, R. D. (2013). How to assess, diagnose, refer and treat adult obstructive sleep apnoea: A commentary on the choices. The Medical Journal of Australia, 199, 21-26. http://dx .doi.org/10.5694/mja13.10909

Marin, J. M., Agusti, A., Villar, I., Forner, M., Nieto, D., Carrizo, S. J., . . . Jelic, S. (2012). Association between treated and untreated obstructive sleep apnea and risk of hypertension. JAMA: Journal of the American Medical Association, 307, 2169-2176. http://dx.doi.org/10.1001/jama .2012 .3418

Marin, J. M., Carrizo, S. J., \& Kogan, I. (1998). Obstructive sleep apnea and acute myocardial infarction: Clinical implications of the association. Sleep: Journal of Sleep Research \& Sleep Medicine, 21, 809-815.

Meade, A. W., Johnson, E. C., \& Braddy, P. W. (2008). Power and sensitivity of alternative fit indices in tests of measurement invariance. Journal of Applied Psychology, 93, 568-592. http://dx.doi.org/10.1037/ 0021-9010.93.3.568

Milfont, T. L., \& Fischer, R. (2010). Testing measurement invariance across groups: Applications in cross-cultural research. International Journal of Psychological Research, 3, 111-130.
Morin, A. J., Arens, A. K., \& Marsh, H. W. (2016). A bifactor exploratory structural equation modeling framework for the identification of distinct sources of construct-relevant psychometric multidimensionality. Structural Equation Modeling, 23, 116-139. http://dx.doi.org/10.1080/ 10705511.2014.961800

Motamedi, K. K., McClary, A. C., \& Amedee, R. G. (2009). Obstructive sleep apnea: A growing problem. The Ochsner Journal, 9, 149-153.

Muthén, L. K., \& Muthén, B. O. (1998-2011). MPlus user's guide (6th ed.). Los Angeles, CA: Author.

Nanthakumar, S., Bucks, R. S., \& Skinner, T. C. (2016). Are we overestimating the prevalence of depression in chronic illness using questionnaires? Meta-analytic evidence in obstructive sleep apnoea. Health Psychology, 35, 423-432. http://dx.doi.org/10.1037/hea0000280

Ng, S. S. S., Chan, T. O., To, K. W., Ngai, J., Tung, A., Ko, F. W. S., \& Hui, D. S. C. (2009). Validation of a portable recording device (ApneaLink) for identifying patients with suspected obstructive sleep apnoea syndrome. Internal Medicine Journal, 39, 757-762. http://dx.doi .org/10.1111/j.1445-5994.2008.01827.x

Norton, P. J. (2007). Depression Anxiety and Stress Scales (DASS-21): Psychometric analysis across four racial groups. Anxiety, Stress, \& Coping: An International Journal, 20, 253-265. http://dx.doi.org/10 $.1080 / 10615800701309279$

Nye, C. D., \& Drasgow, F. (2011). Effect size indices for analyses of measurement equivalence: Understanding the practical importance of differences between groups. Journal of Applied Psychology, 96, 966980. http://dx.doi.org/10.1037/a0022955

Nye, C. D., Roberts, B. W., Saucier, G., \& Zhou, X. (2008). Testing the measurement equivalence of personality adjective items across cultures. Journal of Research in Personality, 42, 1524-1536. http://dx.doi.org/10 .1016/j.jrp.2008.07.004

Peppard, P. E., Young, T., Barnet, J. H., Palta, M., Hagen, E. W., \& Hla, K. M. (2013). Increased prevalence of sleep-disordered breathing in adults. American Journal of Epidemiology, 177, 1006-1014. http://dx .doi.org/10.1093/aje/kws342

Ragette, R., Wang, Y., Weinreich, G., \& Teschler, H. (2010). Diagnostic performance of single airflow channel recording (ApneaLink) in home diagnosis of sleep apnea. Sleep and Breathing, 14, 109-114. http://dx .doi.org/10.1007/s11325-009-0290-2

Schrag, A., Barone, P., Brown, R. G., Leentjens, A. F., McDonald, W. M., Starkstein, S., . . . Goetz, C. G. (2007). Depression rating scales in Parkinson's disease: Critique and recommendations. Movement Disorders, 22, 1077-1092. http://dx.doi.org/10.1002/mds.21333

Schröder, C. M., \& O’Hara, R. (2005). Depression and obstructive sleep apnea (OSA). Annals of General Psychiatry, 4, 13. http://dx.doi.org/10 $.1186 / 1744-859 X-4-13$

Spicuzza, L., Caruso, D., \& Di Maria, G. (2015). Obstructive sleep apnoea syndrome and its management. Therapeutic Advances in Chronic Disease, 6, 273-285.

Steenkamp, J. B. E., \& Baumgartner, H. (1998). Assessing measurement invariance in cross-national consumer research. Journal of Consumer Research, 25, 78-107. http://dx.doi.org/10.1086/209528

Steiger, J. H., \& Lind, J. C. (1980, May). Statistically based tests for the number of common factors. In Proceedings of the Annual Meeting of the Psychometric Society (Vol. 758, pp. 424-453). Iowa City, IA.

Szabó, M. (2010). The short version of the Depression Anxiety Stress Scales (DASS-21): Factor structure in a young adolescent sample. Journal of Adolescence, 33, 1-8. http://dx.doi.org/10.1016/j.adolescence 2009.05.014

Tasali, E., Mokhlesi, B., \& Van Cauter, E. (2008). Obstructive sleep apnea and type 2 diabetes: Interacting epidemics. Chest, 133, 496-506. http:// dx.doi.org/10.1378/chest.07-0828

Tucker, L. R., \& Lewis, C. (1973). A reliability coefficient for maximum likelihood factor analysis. Psychometrika, 38, 1-10. http://dx.doi.org/10 .1007/BF02291170 
Vandenberg, R. J., \& Lance, C. E. (2000). A review and synthesis of the measurement invariance literature: Suggestions, practices, and recommendations for organizational research. Organizational Research Methods, 3, 4-70. http://dx.doi.org/10.1177/109442810031002

Vgontzas, A. N., Bixler, E. O., \& Chrousos, G. P. (2005). Sleep apnea is a manifestation of the metabolic syndrome. Sleep Medicine Reviews, 9, 211-224. http://dx.doi.org/10.1016/j.smrv.2005.01.006

Wood, B. M., Nicholas, M. K., Blyth, F., Asghari, A., \& Gibson, S. (2010). The utility of the short version of the Depression Anxiety Stress Scales (DASS-21) in elderly patients with persistent pain: Does age make a difference? Pain Medicine, 11, 1780-1790. http://dx.doi.org/10.1111/j .1526-4637.2010.01005.x

Yaggi, H. K., Concato, J., Kernan, W. N., Lichtman, J. H., Brass, L. M., \& Mohsenin, V. (2005). Obstructive sleep apnea as a risk factor for stroke and death. The New England Journal of Medicine, 353, 2034-2041. http://dx.doi.org/10.1056/NEJMoa043104 
